\title{
ON RIEMANNIAN MANIFOLDS OF FOUR DIMENSIONS ${ }^{1}$
}

\author{
SHIING-SHEN CHERN
}

Introduction. It is well known that in three-dimensional elliptic or spherical geometry the so-called Clifford's parallelism or parataxy has many interesting properties. A group-theoretic reason for the most important of these properties is the fact that the universal covering group of the proper orthogonal group in four variables is the direct product of the universal covering groups of two proper orthogonal groups in three variables. This last-mentioned property has no analogue for orthogonal groups in $n(>4)$ variables. On the other hand, a knowledge of three-dimensional elliptic or spherical geometry is useful for the study of orientable Riemannian manifolds of four dimensions, because their tangent spaces possess a geometry of this kind. It is the purpose of this note to give a study of a compact orientable Riemannian manifold of four dimensions at each point of which is attached a three-dimensional spherical space. This necessitates a more careful study of spherical geometry than hitherto given in the literature, except, so far as the writer is aware, in a paper by E. Study [2]. ${ }^{2}$ Our main result consists of two formulas, which express two topological invariants of a compact orientable differentiable manifold of four dimensions as integrals over the manifold of differential invariants constructed from a Riemannian metric previously given on the manifold. These two topological invariants have a linear combination which is the Euler-Poincare characteristic.

1. Three-dimensional spherical geometry. We consider an oriented Euclidean space of four dimensions $E^{4}$ with the coordinates $x_{0}, x_{1}, x_{2}, x_{3}$. In $E^{4}$ let $S^{3}$ be the oriented unit hypersphere defined by the equation

$$
x_{0}^{2}+x_{1}^{2}+x_{2}^{2}+x_{3}^{2}=1 .
$$

Three-dimensional spherical geometry is concerned with properties on $S^{3}$ which remain invariant under the rotation group (that is, the proper orthogonal group) of $E^{4}$ leaving the origin fixed.

Received by the editors June 22, 1945.

1 The content of this paper was originally intended to be an illustration in the author's article, Some new viewpoints in differential geometry in the large, which is due to appear in this Bulletin. Later it appeared more advisable to publish these results separately, but a comparison with the above-mentioned article, in particular $\$ 7$, is recommended.

2 Numbers in brackets refer to the references cited at the end of the paper. 
We call a frame an ordered set of four mutually perpendicular unit vectors $e_{0}, e_{1}, e_{2}, e_{3}$. There exists one and only one rotation carrying one frame to another. The coordinates $x_{0}, x_{1}, x_{2}, x_{3}$ of a point $\mathfrak{x} \in S^{3}$ with respect to the frame $e_{0}, e_{1}, e_{2}, e_{3}$ are defined by the equation

$$
\mathfrak{x}=x_{0} \mathfrak{e}_{0}+x_{1} \mathfrak{e}_{1}+x_{2} \mathfrak{e}_{2}+x_{3} \mathfrak{e}_{3} .
$$

Let $e_{0}^{*}, e_{1}^{*}, e_{2}^{*}, e_{3}{ }^{*}$ be a frame related to $e_{0}, e_{1}, e_{2}, e_{3}$ by means of the relations

$$
\mathrm{e}_{\alpha}^{*}=\sum_{\beta=0}^{3} a_{\alpha \beta} \mathrm{e}_{\beta}, \quad \alpha=0,1,2,3,
$$

where $\left(a_{\alpha \beta}\right)$ is a proper orthogonal matrix, and let $x_{0}{ }^{*}, x_{1}{ }^{*}, x_{2}{ }^{*}, x_{3}{ }^{*}$ be the coordinates of the same point $x$ with respect to the frame $\mathrm{e}_{0}^{*}, \mathrm{e}_{1}^{*}, \mathrm{e}_{2}^{*}, \mathrm{e}_{3}^{*}$. Then we have

$$
x_{\alpha}^{*}=\sum_{\beta=0}^{3} a_{\alpha \beta} x_{\beta}, \quad \alpha=0,1,2,3 .
$$

The properties of spherical geometry are those which, when expressed in terms of coordinates with respect to a frame, remain invariant under change of the frame.

Let $x_{0}, x_{1}, x_{2}, x_{3}$ be the coordinates of a point $x$ with respect to a frame $e_{0}, e_{1}, e_{2}, e_{3}$, as defined by (2). To these coordinates we associate a unit quaternion

$$
X=x_{0}+x_{1} i+x_{2} j+x_{3} k, \quad N(X)=1,
$$

where $N(X)$ denotes the norm of $X$. Let

$$
X^{*}=x_{0}^{*}+x_{1}^{*} i+x_{2}^{*} j+x_{3}^{*} k .
$$

Then the following theorem is well known [1]:

THEOREM 1. The proper orthogonal group (3a) can be expressed in the quaternion notation in the form

$$
X^{*}=A X B \text {, }
$$

where $A, B$ are unit quaternions. It contains the two subgroups

$$
\begin{aligned}
& X^{*}=A X, \\
& X^{*}=X B,
\end{aligned}
$$

called the subgroups of left and right translations respectively. A left translation is a right translation when and only when it is $X^{*}= \pm X$.

It is important to give a distinction between the left and right 
translations. We shall show that this is possible when $S^{3}$ is oriented. When $X_{i}, i=1,2,3,4$, are four quaternions, we introduce the notation $\left|X_{1} X_{2} X_{3} X_{4}\right|$ to denote the value of the determinant formed by their components. Then we have the following theorem:

THEOREM 2. For any three quaternions $X, X^{\prime}, A$ the inequalities

$$
\left|X X^{\prime}, A X, A X^{\prime}\right| \leqq 0, \quad\left|X X^{\prime}, X A, X^{\prime} A\right| \geqq 0
$$

hold. Equality sign holds only when $A= \pm 1$ or when $X, X^{\prime}, A$ are linearly dependent.

To prove this theorem we assume the quaternions to be different from zero and hence, without loss of generality, to be unit quaternions. By a change of frame, which does not affect the values of the determinants in question, we can suppose $X=1$. Let

$$
A=a_{0}+a_{1} i+a_{2} j+a_{3} k, \quad X^{\prime}=x_{0}^{\prime}+x_{1}^{\prime} i+x_{2}^{\prime} j+x_{3}^{\prime} k .
$$

We get then

$$
\begin{aligned}
\left|X X^{\prime}, A X, A X^{\prime}\right|= & -\left(a_{1} x_{2}^{\prime}-a_{2} x_{1}^{\prime}\right)^{2}-\left(a_{2} x_{3}^{\prime}-a_{3} x_{2}^{\prime}\right)^{2} \\
& -\left(a_{3} x_{1}^{\prime}-a_{1} x_{3}^{\prime}\right)^{2} \leqq 0, \\
\left|X X^{\prime}, X A, X^{\prime} A\right|= & \left(a_{1} x_{2}^{\prime}-a_{2} x_{1}^{\prime}\right)^{2}+\left(a_{2} x_{3}^{\prime}-a_{3} x_{2}^{\prime}\right)^{2} \\
& +\left(a_{3} x_{1}^{\prime}-a_{1} x_{3}^{\prime}\right)^{2} \geqq 0 .
\end{aligned}
$$

From these equations the theorem follows.

This theorem gives a criterion to distinguish between the left and the right translations. A reversal of orientation of $S^{3}$ interchanges the left and right translations.

We now put

$$
A=a_{0}+a_{1} i+a_{2} j+a_{3} k, \quad B=b_{0}+b_{1} i+b_{2} j+b_{3} k,
$$

and write out in detail the transformations (6a) and (6b):

$$
\begin{array}{r}
\left\{\begin{array}{l}
x_{0}^{*}=a_{0} x_{0}-a_{1} x_{1}-a_{2} x_{2}-a_{3} x_{3}, \\
x_{1}^{*}=a_{1} x_{0}+a_{0} x_{1}-a_{3} x_{2}+a_{2} x_{3}, \\
x_{2}^{*}=a_{2} x_{0}+a_{3} x_{1}+a_{0} x_{2}-a_{1} x_{3}, \\
x_{3}^{*}=a_{3} x_{0}-a_{2} x_{1}+a_{1} x_{2}+a_{0} x_{3},
\end{array}\right. \\
\left\{\begin{array}{l}
x_{0}^{*}=b_{0} x_{0}-b_{1} x_{1}-b_{2} x_{2}-b_{3} x_{3}, \\
x_{1}^{*}=b_{1} x_{0}+b_{0} x_{1}+b_{3} x_{2}-b_{2} x_{3}, \\
x_{2}^{*}=b_{2} x_{0}-b_{3} x_{1}+b_{0} x_{2}+b_{1} x_{3}, \\
x_{3}^{*}=b_{3} x_{0}+b_{2} x_{1}-b_{1} x_{2}+b_{0} x_{3} .
\end{array}\right.
\end{array}
$$


Let $\mathfrak{A}$ and $\mathfrak{B}$ be respectively the matrices of these transformations. The associativity of quaternion multiplication implies that

$$
\mathfrak{U} \mathfrak{B}=\mathfrak{B} \mathfrak{Y} \text {. }
$$

We then define

$$
\mathbb{E}=\left(\begin{array}{l}
e_{0} \\
e_{1} \\
e_{2} \\
e_{3}
\end{array}\right), \quad \mathbb{F} *=\left(\begin{array}{l}
e_{0}^{*} \\
e_{1}^{*} \\
e_{2}^{*} \\
e_{3}^{*}
\end{array}\right) \text {, }
$$

so that $\&$ and ${ }^{*}$ are one-columned matrices whose elements are vectors. From Theorem 1 we see that the rotation (3) can always be written

$$
\mathfrak{E}^{*}=\mathfrak{X} \mathfrak{B} \mathfrak{F}=\mathfrak{B} \mathfrak{Y} \mathfrak{E} \text {. }
$$

We call two frames $\&$ and ${ }^{*}$ left (right) equivalent if

$$
\mathfrak{F}^{*}=\mathfrak{Y}\left(\mathbb{E} \quad\left(\mathfrak{F}^{*}=\mathfrak{B} \mathscr{E}\right) .\right.
$$

It is evident that this equivalence relation is reflexive, symmetric, and transitive. We now introduce as new figures in spherical geometry classes of left (right) equivalent frames and shall call them the left (right) Clifford figures.

With respect to a given frame $\mathbb{E}_{0}$ a left (right) Clifford figure consists of all frames $\mathfrak{A} \mathfrak{B} \mathfrak{E}_{0}$, where $\mathfrak{B}(\mathfrak{U})$ remains fixed and $\mathfrak{A}(\mathfrak{B})$ varies. As its coordinate we can therefore take the matrix $\mathfrak{B}(\mathfrak{U})$, or the corresponding unit quaternion $B(A)$. From Theorem 1 it follows that two quaternions determine the same Clifford figure, when and only when one is the negative of the other. For our purpose we shall, however, make a distinction between the Clifford figures determined by $+B$ and $-B(+A$ and $-A)$. The new figure will then be called an oriented left (right) Clifford figure. With a natural topology the space of all Clifford figures is homeomorphic to the real projective space of three dimensions and the space of all oriented Clifford figures to the three-dimensional sphere.

With a differentiable family of frames we define

$$
\omega_{\alpha \beta}=d \mathfrak{e}_{\alpha} \cdot \mathfrak{e}_{\beta}, \quad \alpha, \beta=0,1,2,3,
$$

where

$$
\omega_{\alpha \beta}+\omega_{\beta \alpha}=0 \text {. }
$$

The differential forms $\omega_{\alpha \beta}$ satisfy the following equations of structure of our spherical space: 


$$
d \omega_{\alpha \beta}=\sum_{\gamma=0}^{3} \omega_{\alpha \gamma} \omega_{\gamma \beta}, \quad \alpha, \beta=0,1,2,3 .
$$

It is easy to verify that when the frames of the family belong to a left equivalence class we have

$$
\phi_{1} \equiv \omega_{01}-\omega_{23}=0, \phi_{2} \equiv \omega_{02}-\omega_{31}=0, \phi_{3} \equiv \omega_{03}-\omega_{12}=0 .
$$

Similarly, when the frames of the family belong to a right equivalence class, we have

$$
\begin{gathered}
\psi_{1} \equiv-\omega_{01}-\omega_{23}=0, \quad \psi_{2} \equiv-\omega_{02}-\omega_{31}=0, \\
\psi_{3} \equiv-\omega_{03}-\omega_{12}=0 .
\end{gathered}
$$

Using the forms $\phi_{i}, \psi_{i}, i=1,2,3$, instead of $\omega_{\alpha \beta}, \alpha, \beta=0,1,2,3$, the equations of structure (15) can be replaced by the equations

$$
\begin{array}{lll}
d \phi_{1}=\phi_{2} \phi_{3}, & d \phi_{2}=\phi_{3} \phi_{1}, & d \phi_{3}=\phi_{1} \phi_{2}, \\
d \psi_{1}=\psi_{2} \psi_{3}, & d \psi_{2}=\psi_{3} \psi_{1}, & d \psi_{3}=\psi_{1} \psi_{2} .
\end{array}
$$

The form $\phi_{1} \phi_{2} \phi_{3}$ is a volume element in the space $R_{l}$ of oriented left Clifford figures and the same is true of the form $\psi_{1} \psi_{2} \psi_{3}$ in the space $R_{r}$ of oriented right Clifford figures. Let $R_{l}, R_{r}$ denote also the fundamental cycles on the manifolds $R_{l}, R_{r}$. We easily find

$$
\int_{R_{l}} \phi_{1} \phi_{2} \phi_{3}=\int_{R_{r}} \psi_{1} \psi_{2} \psi_{3}=2 \pi^{2} .
$$

It follows that if $Z$ is a three-dimensional cycle of $R_{l}$ which is homologous to $m R_{l}$, we shall have

$$
\frac{1}{2 \pi^{2}} \int_{Z} \phi_{1} \phi_{2} \phi_{3}=m
$$

A similar statement holds for $R_{r}$.

2. Riemannian manifolds of four dimensions. Let $M$ be a compact orientable Riemannian manifold of four dimensions. With $M$ as base space is defined the tangent sphere bundle of unit vectors, on each fibre of which operates the rotation group in four variables. The geometry of a fibre of $M$ is the three-dimensional spherical geometry. The construction of the last section defines two new fibre bundles $\mathfrak{F}_{l}$ and $\mathfrak{F}_{r}$ over $M$, namely the bundles of oriented left and right Clifford figures.

The equations of structure of $M$ are 


$$
d \omega_{\alpha \beta}=\sum_{\gamma=0}^{3} \omega_{\alpha \gamma} \omega_{\gamma \beta}+\Omega_{\alpha \beta}, \quad \alpha, \beta=0,1,2,3 .
$$

We put

$$
\phi_{1}=\omega_{01}-\omega_{23}, \quad \phi_{2}=\omega_{02}-\omega_{31}, \quad \phi_{3}=\omega_{03}-\omega_{12},
$$

and

$$
\psi_{1}=-\omega_{01}-\omega_{23}, \quad \psi_{2}=-\omega_{02}-\omega_{31}, \quad \psi_{3}=-\omega_{03}-\omega_{12} .
$$

Equations (21) then become

$$
\begin{aligned}
& d \phi_{1}=\phi_{2} \phi_{3}+\Phi_{1}, \quad d \phi_{2}=\phi_{3} \phi_{1}+\Phi_{2}, \quad d \phi_{3}=\phi_{1} \phi_{2}+\Phi_{3}, \\
& d \psi_{1}=\psi_{2} \psi_{3}+\Psi_{1}, \quad d \psi_{2}=\psi_{3} \psi_{1}+\Psi_{2}, \quad d \psi_{3}=\psi_{1} \psi_{2}+\Psi_{3},
\end{aligned}
$$

where

$$
\begin{array}{lll}
\Phi_{1}=\Omega_{01}-\Omega_{23}, & \Phi_{2}=\Omega_{02}-\Omega_{31}, & \Phi_{3}=\Omega_{03}-\Omega_{12}, \\
\Psi_{1}=-\Omega_{01}-\Omega_{23}, & \Psi_{2}=-\Omega_{02}-\Omega_{31}, & \Psi_{3}=-\Omega_{03}-\Omega_{12} .
\end{array}
$$

Consider the fibre bundle $\mathfrak{F}_{l}$. Each fibre being homeomorphic to a three-dimensional sphere, the characteristic class of $\mathfrak{F}_{l}$ in $M$ is an integral cohomology class $W_{l}$ of dimension four. Similarly, $\mathfrak{F}_{r}$ gives rise also to an integral cohomology class $W_{r}$ of dimension four. By the multiplication of the homology group and the cohomology group, we put

$$
I_{l}=W_{l} \cdot M, \quad I_{r}=W_{r} \cdot M,
$$

which are topological invariants of $M$ (they are in fact invariants of the differential structure of $M$ under consideration). We shall express $I_{l}, I_{r}$ as integrals over $M$ of differential invariants of the Riemannian metric in $M$.

THEOREM 3. Let $M$ be a compact orientable Riemannian manifold of dimension four and let $\Omega_{\alpha \beta}, \alpha, \beta=0,1,2,3$, and $\Phi_{i}, \Psi_{i}, i=1,2,3$, be the curvature forms of the Riemannian metric constructed according to the equations (21), (24). Then the following formulas hold:

$$
\frac{1}{2 \pi^{2}} \int_{M} \Phi_{1}^{2}+\Phi_{2}^{2}+\Phi_{3}^{2}=I_{l}, \quad \frac{1}{2 \pi^{2}} \int_{M} \Psi_{1}^{2}+\Psi_{2}^{2}+\Psi_{3}^{2}=I_{r} .
$$

If $\chi$ is the Euler-Poincaré characteristic of $M$, we have

$$
-I_{l}+I_{r}=8 \chi \text {. }
$$

To prove this theorem we find from (23) 


$$
\begin{aligned}
d\left(\phi_{1} \phi_{2} \phi_{3}\right) & =\Phi_{1} \phi_{2} \phi_{3}+\Phi_{2} \phi_{3} \phi_{1}+\Phi_{3} \phi_{1} \phi_{2}, \\
d\left(\sum_{i=1}^{3} \phi_{i} \Phi_{i}\right) & =-\left(\Phi_{1} \phi_{2} \phi_{3}+\Phi_{2} \phi_{3} \phi_{1}+\Phi_{3} \phi_{1} \phi_{2}\right)+\sum_{i=1}^{3} \Phi_{i}^{2},
\end{aligned}
$$

which gives

$$
d\left(\phi_{1} \phi_{2} \phi_{3}+\sum_{i=1}^{3} \phi_{i} \Phi_{i}\right)=\sum_{i=1}^{3} \Phi_{i}^{2}
$$

By a standard procedure in the theory of fibre bundles we see that it is always possible to define a mapping of $M$ into $\mathfrak{F}_{l}$, with a finite number of singular points, such that the image of a point $P$ of $M$ is an oriented left Clifford figure attached to that point. The sum of the indices at the singular points is equal to $I_{l}$. The first formula in (26) then follows immediately from (20) and (28). A similar argument applies to the second formula.

From (26) we get, by subtraction,

$$
I_{l}-I_{r}=-\frac{2}{\pi^{2}} \int_{M} \Omega_{01} \Omega_{23}+\Omega_{02} \Omega_{31}+\Omega_{03} \Omega_{12} .
$$

By the formula of Allendoerfer-Weil we have

$$
\chi=\frac{1}{4 \pi^{2}} \int_{M} \Omega_{01} \Omega_{23}+\Omega_{02} \Omega_{31}+\Omega_{03} \Omega_{12} .
$$

Consequently, we have

$$
-I_{l}+I_{r}=8 \chi
$$

Hence the theorem is proved.

The theorem shows that by the consideration of the fibre bundles $\mathfrak{F}_{l}, \mathfrak{F}_{r}$ over $M$ we have introduced, besides the Euler-Poincaré characteristic, essentially one new topological invariant of $M$. We denote this invariant by

$$
\eta=\left(I_{l}+I_{r}\right) / 2
$$

which is an integer. Then we have

$$
2 \pi^{2} \eta=\int_{M} \Omega_{01}^{2}+\Omega_{02}^{2}+\Omega_{03}^{2}+\Omega_{12}^{2}+\Omega_{23}^{2}+\Omega_{31}^{2} .
$$

If $M$ is the toroidal space, then $\eta=0$, for a locally Euclidean Riemannian metric can then be defined in $M$. If $M$ is the four-dimensional sphere, the induced metric of $M$ obtained by imbedding it as 
a unit hypersphere in the five-dimensional Euclidean space is of constant positive Riemannian curvature. We have then

$$
\Omega_{\alpha \beta}=-\omega_{\alpha} \omega_{\beta}, \quad \alpha, \beta=0,1,2,3 .
$$

It follows that $\eta(M)=0$, if $M$ is a sphere.

\section{REFERENCES}

1. F. Klein, Vorlesungen uber nicht-Euklidischen Geometrie, pp. 238-241.

2. E. Study, Beiträge zur nicht-Euclidischen Geometrie, Amer. J. Math. vol. 29 (1907) pp. 116-159.

Institute for Advanced Study AND

TSING Hua University 